\title{
Tricuspid valve disease
}

\section{Background}

The tricuspid valve of the heart is situated between the upper and lower chambers of the right side of the heart, known as the right atrium and right ventricle respectively. It opens and closes in a coordinated fashion to ensure that blood flows in a forward direction from the right atrium to the right ventricle. This enables blood to be pumped efficiently from the right side of the heart to the lungs for adequate oxygenation. Tricuspid valve disease occurs when this function is impaired. The more common form of tricuspid disease, known as tricuspid regurgitation or insufficiency, occurs when the valve cannot close adequately, resulting in the backward leakage of blood into the right atrium with each heart contraction. Alternatively, the valve may become stiff and narrowed preventing the forward flow of blood from the atrium to the ventricle, known as tricuspid stenosis. If severe, surgical repair or replacement of the valve may be necessary to relieve symptoms, prevent complications, prolong survival and improve one's quality of life.

\section{Causes}

There are a numerous causes of tricuspid valve disease. Tricuspid regurgitation most commonly occurs in conditions which cause the right ventricle to expand or dilate, resulting in enlargement of the valve's ring-like base. These conditions include heart failure, which may be a cause and consequence of tricuspid valve disease. Heart failure itself may be caused by pathology of the right or left side of the heart, for example coronary artery disease, left-sided valvular disease and conditions which affect the muscle of the heart, known as cardiomyopathies. Tricuspid regurgitation may also result from ventricular dilation secondary to high blood pressure in the lung circulation, known as pulmonary hypertension, which may be caused by left-sided heart disease or lung disease, such as emphysema. Less common causes include trauma, infective endocarditis, rheumatic heart disease, carcinoid syndrome, degeneration of the valve's supporting connective tissue and congenital heart defects. Tricuspid stenosis most commonly results from rheumatic heart disease, which causes the leaflets of the valve to become thick, hardened and less able to open widely, thus restricting forward blood flow.

\section{Signs and symptoms}

Tricuspid valve disease may not cause any symptoms until it has become severe. When individuals are symptomatic, they may report fatigue, shortness of breath, decreased exercise tolerance, swelling of the legs or abdomen and a pulsing sensation in the neck. Affected individuals may be noted to have a heart murmur, an enlarged, pulsating liver as well as a pulsating neck vein, reflecting the backflow of blood from the right side of the heart.

\section{Diagnosis}

The most common test to diagnose tricuspid valve disease is an echocardiogram, which provides detailed ultrasound imaging of the heart. The sound device or transducer may be placed on the chest wall, known as a transthoracic echocardiogram, or a tube and transducer are inserted into the esophagus for more detailed imaging, known as a transesophageal echocardiogram. These tests evaluate the structure and function of both the heart muscle and valves. Other tests that may be carried out include an electrocardiogram, whereby electrodes assess the heart's electrical activity, and stress tests, which assess the response of the heart to physical exertion, either by electrocardiography or echocardiography.

\section{Management}

Management of tricuspid valve disease depends on the severity of the disease and symptoms as well as the underlying cause. Certain medications may be used in the treatment of underlying heart failure both to relieve symptoms and prolong survival. Eligibility for surgery will be evaluated by your surgeon based on symptoms, the severity of tricuspid disease as well as the extent to which it has resulted in heart failure. Options for surgery include tricuspid valve repair or replacement. Repair may involve insertion of a synthetic 'annuloplasty' ring to tighten and stabilize the existing valve. Valve replacement involves removal of the damaged valve and insertion of a new biological or mechanical valve, the latter requiring life-long treatment with blood-thinning medication to prevent formation of blood clots on the valve. These surgical options may now be performed by minimally invasive approaches including roboticassisted techniques. Tricuspid surgery may significantly alleviate symptoms, enhance one's quality of life and prolong survival. As with all operations there are risks of bleeding, infection, formation of blood clots, prolonged pain following the operation and the possibility of a reoperation. As always, the risks should be weighed against the benefits and discussed with your surgeon. For more information, please visit the following websites:

https://my.clevelandclinic.org/health/articles/tricuspid-valve-disease http://www.mayoclinic.org/diseases-conditions/tricuspid-valveregurgitation/home/ovc-20120489

http://ctsurgerypatients.org/adult-heart-disease/tricuspid-valve-disease

doi: 10.21037/acs.2017.05.01

\begin{tabular}{l}
\hline Section Writer: Christopher Harris \\
\hline Illustration Editor: Beth Croce \\
\hline Section Editor: Stine Munkholm-Larsen \\
\hline
\end{tabular}

For specific information concerning your medical condition, ACS suggests that you consult your physician. This page may be photocopied non-commercially by physicians to share with patients. 